\title{
Pode a correção cirúrgica de cardiopatias pediátricas e congênitas conviver com baixa mortalidade?: revisão de 10 anos de experiência com 1088 cirurgias
}

Danton R. da Rocha LOURES*, Paulo Roberto BROFMAN*, Edison José RIBEIRO*, Paulo Roberto F. ROSSI*, Marcos Augusto Alves PEREIRA*, Antoninho KRICHENKO*, Ronaldo da Rocha Loures BUENO*, Alexandre VARELA*, Victor BAUER ${ }^{\star}$, Maria João F. AMORIM*, Lauro J. C. LINHARES ${ }^{\star}$, Edimara F. SEEGMUL.LER*, Nelson MOZACHI*

LOURES, D. R. R.; BROFMAN, P. R; RIBEIRO, E. J.; ROSSI, P. R.; PEREIRA, M. A. A.; KRICHENKO, A.; BUENO, R. R. L.; VARELA, A.; BAUER, V.; AMORIM, M. J. F.; LINHARES, L. J. C.; SEEGMULLER, E. F.; MOZACHI, N. - Pode a correção cirúrgica de cardiopatias pediátricas e congênitas conviver com baixa mortalidade?: revisão de 10 anos de experiência com 1088 cirurgias. Rev. Bras. Circ. Cardiovasc., 2(1):32-41, 1987

RESUMO: Em um período de 10 anos, de novembro de 1976 a novembro de 1986, foram realizadas 1088 cirurgias, em pacientes com cardiopatias pediátricas, com menos de 15 anos de idade, e em pacientes com cardiopatias congênitas. Foram corrigidos, com o auxilio da CEC, 670 casos e, com cirurgia clássica, 418 casos. Foram reoperados 111 pacientes $(10,2 \%)$. Pacientes com lesōes valvares adquiridas e com menos de 15 anos de idade, analisados neste trabalho, compreenderam 120 casos $(11,1 \%)$. A mortalidade hospitalar global foi de $10,4 \%$, sendo $11,2 \%$ com auxilio de CEC e $9,1 \%$ sem CEC. Foi observada uma queda de mortalidade, nos anos de 1985 e 1986, correspondendo a 6,8\% e 5,7\%, respectivamente. Nesse período de 2 anos, foram realizadas 179 cirurgias, sendo corrigidas 54 cardiopatias congênitas cianóticas, com 7 óbitos (12,9\%), e 125 acianóticas, com 2 óbitos (1,6\%). A maior mortalidade ocorreu no primeiro ano de vida $(20,7 \%)$, havendo uma diminuição do número de óbitos nas outras faixas etárias, especialmente acima do quarto ano de vida. A análise dos fatores que influíram na diminuição da mortalidade revelou: indicaçâo mais freqüente de cirurgias pediátricas no primeiro ano de vida, análise cuidadosa da anatomia cirúrgica, definições precisas do momento da intervenção e técnicas de cirurgia, melhor proteção miocárdica, condiçōes físicas e médicas no pós-operatório e maior experiência cirúrgica.

DESCRITORES: cardiopatias pediátricas, cirurgia; cardiopatias congênitas, cirurgia.

\section{INTRODUÇÃO}

Em um período de 10 anos, de novembro de 1976 a novembro de 1986, foram realizadas 1088 cirurgias em pacientes com cardiopatias pediátricas adquiridas, com menos de 15 anos de idade e em pacientes com cardiopatias congênitas. A cir- culação extracorpórea (CEC) foi empregada em 670 casos e a cirurgia plástica, em 418 . Foram reoperados, nesse período, 111 pacientes $(10,2 \%)$. Lesões valvares adquiridas, na faixa pediátrica para análise neste trabalho, corresponderam a 120 casos $(11,1 \%)$ (Tabela 1$)$.

\footnotetext{
Trabalho realizado no Hospital Evangélico de Curitiba. Curitiba, PR, Brasil.

Apresentado ao 14: Congresso Nacional de Cirurgia Cardiaca, Salvador, BA, 27 e 28 de març, 1987

- Do Hospital Evangélico de Curitiba.

Endereço para separatas: Danton R. Loures. Rua Augusto Stellfeld, 2088 - 4! andar. 80000 Curitiba, PR, Brasil.
} 
LOURES, D. R.; BROFMAN, P. R.: RIBEIRO, E. J.; ROSSI, P. R. F.; PEREIRA, M. A. A.: KRICHENKO, A.; BUENO, R. R. L.; VARELA, A.; BAUER, V.; AMORIM, M. J. F.; LINHARES, L. J. C.; SEEGMULLER, E. F.; MOZACHI, N. - Pode a correção cirúrgica de cardiopatias pediátricas e congênitas conviver com baixa mortalidade?: revisão de 10 anos de experiência com 1088 cirurgias. Rev. Bras. Cir. Cardiovasc., 2(1):32-41, 1987

\section{TABELA 1}

EXPERIENCIA GLOBAL NO TRATAMENTO CIRÚRGICO DAS CARDIOPATIAS PEDIÁTRICAS E OU CONGENITAS - 10 ANOS

\begin{tabular}{|c|c|c|c|c|c|c|c|c|c|c|c|c|}
\hline \multirow{4}{*}{$\begin{array}{l}\text { CEC } \\
670 / 75=11,2 \%\end{array}$} & \multirow{2}{*}{ Congênita } & 1977 & 1978 & 1979 & 1980 & 1981 & 1982 & 1983 & 1984 & 1985 & 1986 & Total \\
\hline & & $60 / 7$ & $55 / 8$ & $80 / 14$ & $50 / 7$ & $65 / 7$ & $55 / 5$ & $63 / 8$ & $36 / 6$ & $34 / 3$ & $52 / 2$ & $550 / 67=12,2 \%$ \\
\hline & Valvular & $8 / \cdot$ & $9 / 2$ & $10 / 2$ & $16 / 2$ & $10 / 1$ & $15 / 1$ & $12 \%$ & $18 \%$ & $13 /$. & 9\%. & $120 / 8=6.7 \%$ \\
\hline & Total & $68 / 7$ & $64 / 10$ & $90 / 16$ & $66 / 9$ & $75 / 8$ & $70 / 6$ & $75 / 8$ & $54 / 6$ & $47 / 3$ & $61 / 2$ & $670 / 75=11,2 \%$ \\
\hline \multirow{4}{*}{$\begin{array}{l}\text { SICEC } \\
418 / 38=9,1 \%\end{array}$} & Congênita & $7 / 5$ & $31 / 1$ & $41 / 5$ & $35 / 4$ & $50 / 6$ & $30 / 3$ & $29 / 5$ & $28 / 3$ & $25 / 2$ & $31 / 4$ & $307 / 38=12,3 \%$ \\
\hline & Reoperados & $28 / 5$ & $15 \%$ & $12 / \cdot$ & $15 /$. & 9/. & $10 \%$ & $3 \%$ & 4/. & थ. & $13 \%$ & $111 \% .0 \%$ \\
\hline & Total & $35 / 5$ & $46 / 1$ & $53 / 5$ & $50 / 4$ & $59 / 6$ & $40 / 3$ & 3215 & $32 / 3$ & $27 / 2$ & $44 / 4$ & $418 / 38=9,1 \%$ \\
\hline & & $103 / 12$ & $110 / 11$ & $143 / 21$ & $116 / 13$ & $134 / 14$ & $110 / 9$ & $107 / 13$ & $86 / 9$ & $74 / 5$ & $105 / 6$ & \multirow{2}{*}{$\begin{array}{c}\text { Total geral: } \\
1088 / 113 \\
10,4 \%\end{array}$} \\
\hline & & 11,6 & $10 \%$ & $14,7 \%$ & $11,3 \%$ & $10,4 \%$ & $8,2 \%$ & $12,1 \%$ & $10,5 \%$ & $6,8 \%$ & $5,7 \%$ & \\
\hline
\end{tabular}

$\mathrm{CEC}=$ circulação extracorpórea

$\mathrm{S} / \mathrm{CEC}=$ sem $\mathrm{CEC}$

№/NNo = número de casos operados/números de óbitos

A mortalidade hospitalar global foi de $10,4 \%$, sendo sem e com CEC, respectivamente, $9,1 \%$ e $11,2 \%$.

No período de 1985 e 1986, a mortalidade cirúrgica apresentou uma nítida diminuição em relação aos anos anteriores e à média global, correspondendo a $6,8 \%$ e $5,7 \%$, respectivaente

No decorrer destes 10 anos de experiência, houve diversas modificações do atendimento médico, nos períodos de pré, trans e pós-operatório, e se coloca em destaque a investigação diagnóstica, o preparo dos pacientes, as definições precisas do momento da intervenção e técnicas cirúrgicas, a proteção miocárdica, a anestesia, a maior experiência cirúrgica, melhor assistência respiratória mecânica, o atendimento médico de enfermagem no pós-operatório imediato e a criação de uma unidade de terapia intensiva pediátrica.

O presente trabalho procura analisar esta experiência e identificar os fatores que contribuíram para menor mortalidade e morbidade.

\section{MATERIAL E MÉTODO}

Foram realizadas, no período de novembro de 1976 a novembro de 1986, 1088 correções cirúrgicas pediátricas em pacientes com menos de 15 anos de idade e em pacientes com cardiopatias congênitas em todas as idades.

Esta experiência de 10 anos foi analisada, anualmente, em quatro grupos: grupo $\mathrm{A}$ - corrigidas com CEC; grupo B - corrigidas sem circulação extracorpórea; grupo C - reoperaçōes; grupo D - valvares com menos de 15 anos de idade.
Nos anos de 1985 e 1986, para melhor compreender seus resultados, os grupos $\mathrm{A}, \mathrm{B} \mathrm{e} \mathrm{C}$ foram subdivididos em cardiopatia cianótica e acianótica e em grupos etários ( $<1$ ano, 1 a 4 anos, 4 a 15 anos e $>15$ anos).

No protocolo de investigação destas cardiopatias, estão incluídos os seguintes exames laboratoriais: raio $X$ de tórax, eletrocardiograma, perfil metabólico e sangüíneo, ecocardiografia mono e bidimensional e estudo hemodinâmico.

A proteção miocárdica sofreu, nestes 10 anos, diversas modificações. Inicialmente, se fazia apenas reperfusão cada 15 minutos e normotermia. $\mathrm{Na}$ segunda fase, foi empregada a hipotermia moderada. Na terceira fase, foi acrescentada solução gelada no saco pericárdico e, a partir de 1983 , a solução cardioplégica cristalóide gelada a $4^{\circ} \mathrm{C}$ entrou no protocolo, em associação com hipotermia moderada e solução de Shumway.

No período de pós-operatório, os pacientes foram orientados por intensivistas e pediatra-cardiologistas, atendidos por enfermagem especializada em crianças, em uma unidade de terapia intensiva pediátrica.

Em alguns casos de recém-natos com cardiopatias congênitas cianóticas, o estudo hemodinâmico foi dispensado e a correção cirúrgica, geralmente paliativa, foi baseada apenas nas informações da ecocardiografia módulo $\mathrm{M}$ e bidimensional.

A análise desta experiência foi dividida em:

1) Avaliação anual;

2) Divisão por cardiopatias corrigidas com CEC, sem CEC, reoperações e valvopatias. 
LOURES, D. R.; BROFMAN, P. R.; RIBEIRO, E. J.; ROSSI, P. R. F.; PEREIRA, M. A. A.; KRICHENKO, A.; BUENO, R. R. L.; VARELA, A.; BAUER, V.; AMORIM, M. J. F.; LINHARES, L. J. C.; SEEGMULLER, E. F.; MOZACHI, N. - Pode a correção cirúrgica de cardiopatias pediátricas e congênitas conviver com baixa mortalidade?; revisão de 10 anos de experiência com 1088 cirurgias. Rev. Bras. Cir. Cardiovasc., 2(1):32-41, 1987.

A experiência dos dois últimos anos (1985 e 1986) foi analisada e dividida em: cardiopatias cianóticas e acianóticas; corrigidas antes de 1 ano, entre 1 e 4 anos, entre 4 e 15 anos e acima de 15 aos de vida.

Correção da tetralogia de Fallot, defeito do septo atrioventricular e valvopatias foram submetidos a uma análise pormenorizada.

\section{RESULTADOS}

Foram realizadas 1088 cirurgias em pacientes com cardiopatias em fase pediátrica (menos de 15 anos de idade) e/ou cardiopatias congênitas em todas as idades. A mortalidade cirúrgica hospitalar foi de $10,4 \%$. Houve uma nítida diminuição na mortalidade, nos anos de 1985 e 1986, sendo, respectivamente, de 6,8 e $5,7 \%$.

Foram realizadas 857 cirurgias para correção de cardiopatias congênitas $(78,9 \%)$, sendo corrigidas com CEC em 550 casos e através de cirurgia plástica em 307 casos.

As valvopatias operadas em pacientes com menos de 15 anos de idade e analisadas neste trabaIho corresponderam a 120 casos, com 8 obitos $(6,7 \%)$.

A incidência de reoperações, no período hospitalar, foi de $10,2 \%$. Neste cálculo, foram consideradas somente as reoperações ocorridas no período hospitalar.

As reoperações mais freqüentes, no período de 1985 e 1986 , foram relacionadas a sangramento e a arritmias (Tabela 2).

Dentre as cardiopatias congênitas cianóticas corrigidas com CEC, $76,2 \%$ foram de tetralogia de Fallot e representaram $13,1 \%$ da estatística global (143/1088 casos). No primeiro ano de vida, a cirurgia paliativa foi a opção de escolha em $90,1 \%$.
A mortalidade, neste grupo, foi de $20,0 \%$. A correção definitiva foi a escolha mais freqüente, após o 1 : ano de vida ( $127 / 132$ casos, $96,2 \%)$.

A mortalidade foi menor a partir do quarto ano, $12,1 \%$ e $3,7 \%$, correspondendo, respectivamente, aos grupos etários de 4 a 15 anos e acima de 15 anos de idade (Tabela 3 ).

\section{TABELA 2}

INCIDENNCIA DE REOPERACÖES HOSPITALARES NOS ANOS DE 1985 E 1986

\begin{tabular}{|l|c|}
\hline \multicolumn{2}{|l|}{ Causas de reoperações de 1985/1986 } \\
\hline Sangramento & 5 \\
\hline Tamponamento & 2 \\
\hline Bloqueio AV total & 3 \\
\hline Hiperfluxo do BT & 1 \\
\hline Hipofluxo do BT & 1 \\
\hline Diástase esternal & 2 \\
\hline Corpo estranho (CAE) & 1 \\
\hline Total & $15 / 179$ casos $=8,4 \%$ \\
\hline
\end{tabular}

BT = anastomose de Blalock - Taussig

$\mathrm{CRE}=$ catéter de átrio esquerdo

Dentre as cardiopatias de hiperfluxo pulmonar, o defeito do septo átrio-ventricular (DSAV) é analisado em detalhes (Tabela 4). Foram corrigidos 29 casos de DSAV, sendo 17 do tipo parcial e 12 total. A mortalidade global foi de $17,2 \%$. Não houve óbito no grupo de DSAV parcial e houve uma mortalidade de $41,7 \%$ na DSAV total. No grupo da DSAV total, os 6 últimos casos foram realizados sem mortalidade em uma experiência acumulada em apenas dois anos. A idade variou de 10 meses a 45 anos, com idade média, para a DSAV parcial, de 14,2 anos e, para a DSAV total, de 3,2 anos. Dez dos 12 casos de DSAV total foram operados nos primeiros 36 meses, com média de 15,5 meses de vida. 0 sexo feminino foi ligeiramente predominante $(58,6 \%)$. A correção do DSAV parcial consistiu, geralmente, em fechamento da comunicação intera-

TABELA 3

TRATAMENTO CIRÚRGICO DA TETRALOGIA DE FALLOT - EXPERIENCIA DE 10 ANOS

\begin{tabular}{|l|c|c|c|c|c|}
\hline & $<1$ ano & $1-4$ anos & $4-15$ anos & $>15$ anos & Total \\
\cline { 2 - 6 } & $10 / 2$ & $2 / 1$ & $2 / 1$ & $1 /-$ & $15 / 4=26,1 \%$ \\
\cline { 2 - 6 } $\begin{array}{l}\text { Cirurgia paliativa } \\
\text { Cirurgia definitiva }\end{array}$ & $1 /-$ & $45 / 7$ & $56 / 6$ & $26 / 1$ & $128 / 14=10,9 \%$ \\
\hline \multirow{2}{*}{ Total } & $11 / 2$ & $47 / 7$ & $58 / 7$ & $27 / 1$ & $143 / 18$ \\
& $(18,2 \%)$ & $(17,0 \%)$ & $(12,0 \%)$ & $(3,7 \%)$ & $(12,6 \%)$ \\
\hline
\end{tabular}


LOURES, D. R.; BROFMAN, P. R.; RIBEIRO, E. J.; ROSSI, P. R. F.; PEREIRA, M. A. A.; KRICHENKO, A.; BUENO, R. R. L.; VARELA, A.; BAUER, V.; AMORIM, M. J. F.; LINHARES, L. J. C.; SEEGMULLER, E. F.; MOZACHI, N. - Pode a correção cirúrgica de cardiopatias pediátricas e congênitas conviver com baixa mortalidade?: revisão de 10 anos de experiência com 1088 cirurgias. Rev. Bras. Cir. Cardiovasc., 2(1):32-41, 1987.

TABELA 4

TRATAMENTO CIRÚRGICO DO DEFEITO DO SEPTO ATRIOVENTRICULAR (29 CASOS) - EXPERIENCIA DE 10 ANOS

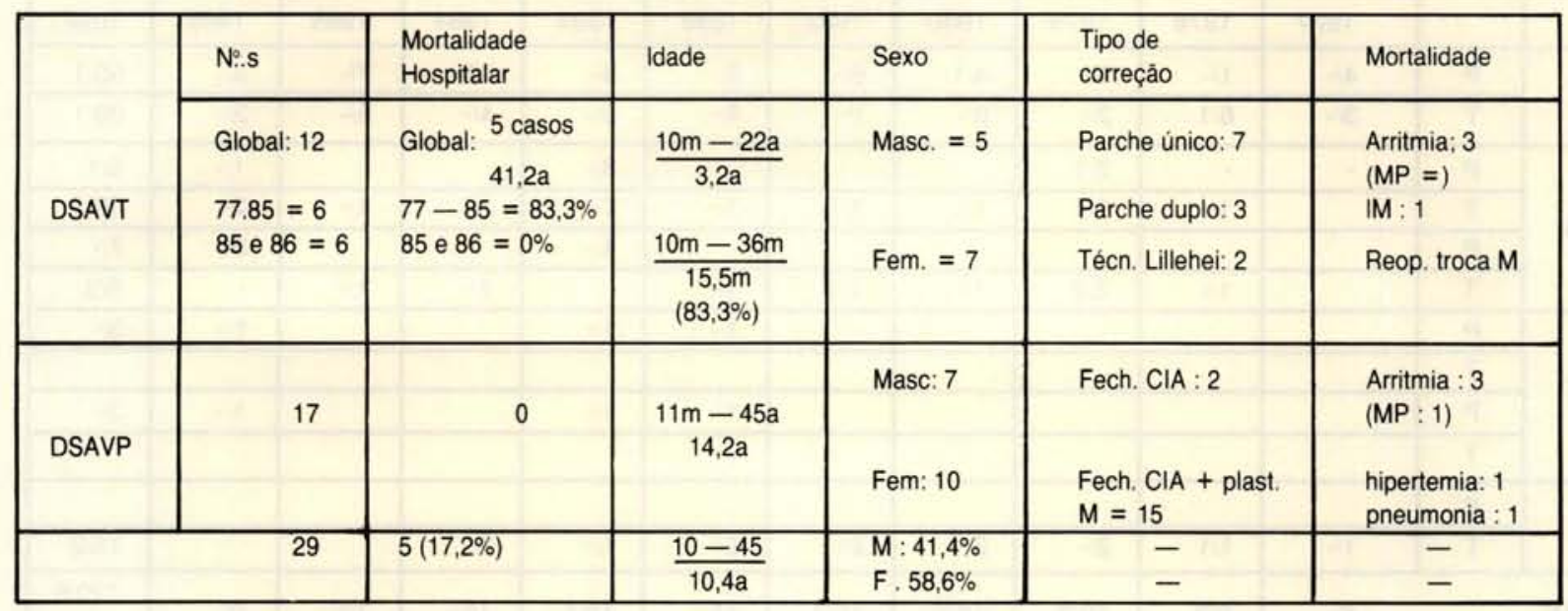

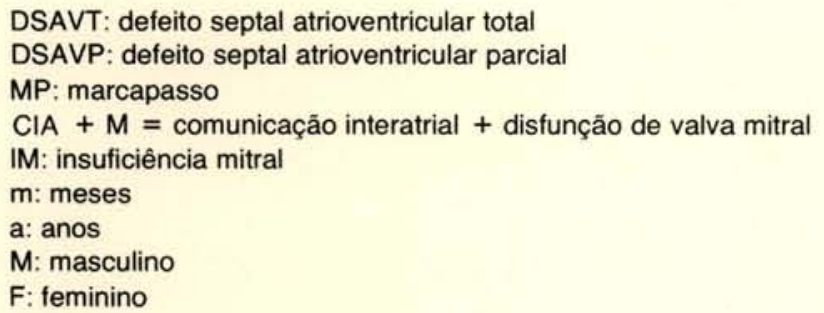

trial (CIA) e plastia da valva mitral $(88,2 \%)$. A correção do DSAV total consistiu no emprego de parche único em 7 casos, parche duplo em 3 e, em 2 casos, a comunicação interventricular (CIV) foi corrigida com pontos separados aproximando os bordos da CIV e a valva atrioventricular (LILEHEI et $a\left(i i^{8}\right)$. As complicações mais freqüentes foram com as arritmias (6 casos), sendo implantado marcapasso definitivo em 2 casos (Tabela 4).

$\mathrm{Na}$ experiência global de 1088 cirurgias, as valvopatias em fase pediátrica foram operadas em 120 casos, correspondendo a $11,1 \%$ do global (Tabela 5). A mortalidade, neste grupo, foi de $6,7 \%$, sendo $13,2 \%$ nos primeiros anos e $1,6 \%$ nos 5 últimos e não havendo óbito nos anos de 1983 a 1986. A valva mitral foi tratada em 107 dos 120 $(89,2 \%)$, sendo isoladamente em 88 casos $(73,3 \%)$, associada à valva aórtica em 16 casos $(13,3 \%)$ e à valva tricúspide em 3 casos $(2,5 \%)$. A valva mitral foi tratada de maneira conservadora (comissurotomia, papilotomia, encurtamento de lascínea, anuloplastia e implante de anel de Carpentier), em $59 \operatorname{casos}^{2}$. Esta plástica foi realizada na valva mitral isoladamente em $84,7 \%$ e, em associação com ou- tras valvas, em 15,3\%. A substituição da valva mitral foi necssária em 48 casos, sendo $20,8 \%$ em associação com outras valvas. Inicialmente, as biopróteses de dura-máter foram implantadas. Mas as próteses mecânicas foram o substituto de escoIha nos últimos 7 anos. A valva aórtica foi tratada em 29 casos, sendo isolada em $13 \mathrm{e}$, em associação com a valva mitral, em 16 casos. A valva tricúspide foi tratada em associação com a valva mitral, de maneira conservadora, em 3 casos.

Nos anos de 1985 e 1986, foram operados 28 pacientes com valvopatias, sendo 22 de origem reumática e de origem congênita e/ou em associação com outros defeitos congênitos (Tabela 6). Não houve mortalidade, neste grupo.

O tratamento valvar associado a outras malformações congênitas correspondeu a 6 de 28 casos $(21,4 \%)$ (Tabela 7$)$. Os casos números 2 e 6 da Tabela 7 foram operados em caráter de emergência. No caso n: 2, houve rotura do seio de Valsalva da valva aórtica. No caso n: 6 , o paciente foi operado na vigência de endocardite infecciosa comprometendo as valvas aórticas e pulmonares. Ambas foram trocadas por próteses artificiais. 
LOURES, D. R.; BROFMAN, P. R.; RIBEIRO, E. J.; ROSSI, P. R. F.; PEREIRA, M. A. A.; KRICHENKO, A.; BUENO, R. R. L.; VARELA, A.; BAUER, V.; AMORIM, M. J. F.; LINHARES, L. J. C.; SEEGMULLER, E. F.; MOZACHI, N. - Pode a correção cirúrgica de cardiopatias pediátricas e congênitas conviver com baixa mortalidade?: revisão de 10 anos de experiência com 1088 cirurgias. Rev. Bras. Cir. Cardiovasc., 2(1):32-41, 1987.

\section{TABELA 5}

CORREÇÃO CIRÚRGICA DE VAL VOPATIAS EM FASE PEDIÁTRICA (< 15 ANOS) - EXPERIÊNCIA DE 10 ANOS

\begin{tabular}{|c|c|c|c|c|c|c|c|c|c|c|c|c|}
\hline & & 1977 & 1978 & 1979 & 1980 & 1981 & 1982 & 1983 & 1984 & 1985 & 1986 & Total \\
\hline \multirow{2}{*}{ M } & $P$ & 4/- & $1 /-$ & 3/- & $4 / 1$ & $6 /-$ & 8/- & 3/- & $10 /-$ & 7\%. & 3/- & $50 / 1$ \\
\hline & $T$ & 3/- & $6 / 1$ & $2 /-$ & $8 /-$ & $1 /-$ & 4/- & $3 /-$ & $4 /-$ & $5 /-$ & $2 /-$ & $38 / 1$ \\
\hline \multirow{4}{*}{ MA } & $P$ & - & - & $2 / 1$ & & & & 3/- & & & 1/- & $6 / 1$ \\
\hline & $T$ & & $1 /-$ & $1 / 1$ & $1 /-$ & $1 / 1$ & $1 /-$ & $1 /-$ & $1 /-$ & $1 /-$ & 2/- & $10 / 2$ \\
\hline & $P$ & & & & & & & 4/- & & & 3/- & $7 /-$ \\
\hline & $T$ & & $1 /-$ & $3 / 2$ & $1 /-$ & $1 / 1$ & $1 /-$ & - & $1 /-$ & $1 /-$ & - & $9 / 3$ \\
\hline \multirow{4}{*}{ MT } & $P$ & & & & & & $1 /-$ & $1 /-$ & & & $1 /-$ & 3/- \\
\hline & $T$ & & & & & & & & & & & \\
\hline & $P$ & & & & & & $1 /-$ & $1 /-$ & & & $1 /-$ & 3/- \\
\hline & $T$ & & & & & & & & & & & \\
\hline \multirow{2}{*}{ Ao } & $P$ & & & & & & & & & & & \\
\hline & $\mathrm{T}$ & $1 /-$ & $1 / 1$ & $2 /-$ & $2 / 1$ & $2 /-$ & $2 /-$ & $1 /-$ & 3/- & & & $13 / 2$ \\
\hline \multicolumn{2}{|c|}{ Total } & 8/- & 9/2 & $10 / 2$ & $16 / 2$ & $10 / 1$ & $15 /-$ & $12 / 1$ & $18 /-$ & $13 /-$ & 9/- & $\begin{array}{l}120 / 8 \\
6,7 \%\end{array}$ \\
\hline
\end{tabular}

$$
\begin{aligned}
& M=\text { mitral } \\
& M T=\text { mitro-tricuspídeo } \\
& M A=\text { mitro-aórtico } \\
& A 0=\text { aórtico } \\
& P=\text { plastia } \\
& T=\text { troca valvar }
\end{aligned}
$$

Nos anos de 1985 e 1986, a mortalidade diminuiu em relação à média global (Tabela 8 ). Esta experiência foi analisada por grupos etários, tipo de cardiopatia e tipo de cirurgia. Foram realizadas $16,3 \%$ das cirurgias no 1 : ano de vida e a maior concentração cirúrgica esteve no grupo etário dos 4 a 15 anos. Nestes dois anos, foram realizadas 168 cirurgias, com mortalidade de $6,2 \%$. Foram corrigidas 54 cardiopatias congênitas cianóticas, com 7 óbitos (12,9\%), e 124 cianóticos, com 2 óbitos $(1,6 \%)$. A maior mortalidade esteve, especialmente, acima do quarto ano $(1,7 \%)$.

\section{COMENTÁRIOS}

Nesta experiência de 1088 casos de cirurgia pediátrica (pacientes com menos de 15 anos de idade), e congênitas, foi analisado apenas o período hospitalar. O acompanhamento tardio foi excluído, neste trabalho, devido à impossibilidade de se fazer um seguimento preciso.

A mortalidade hospitalar global desta experiência foi semelhante à de outros Serviços (BINET ${ }^{1}$, em 1985).
Foram consideradas como reoperações aquelas decorrentes diretamente da cirurgia recentemente realizada. A sua incidência aparentemente elevada $(10,2 \%)$ pode ser interpretada como tradução da fragilidade e instabilidade próprias das crianças.

\section{TABELA 6}

\section{CORREÇAO CIRÚRGICA DE VAL VOPATIAS EM FASE} PEDIATRICA ( 15 ANOS)

\section{EXPERIENCIA DE 1985 E 1986}

\begin{tabular}{|l|ll|l|l|}
\hline & \multicolumn{1}{|c|}{1985} & 1986 & Total \\
\hline \multirow{4}{*}{ Mitral } & \multicolumn{1}{|c|}{ plástica + anel Carpentier4 } & 2 & 6 \\
\cline { 2 - 5 } & plástica simples & 3 & 1 & 4 \\
\cline { 2 - 5 } & troca valvar & 5 & 2 & 7 \\
\cline { 2 - 5 } & dupla troca mecânica & 1 & - & 1 \\
\cline { 2 - 5 } M.A. & troca M + P/Ao & - & 2 & 2 \\
\cline { 2 - 5 } & plástica + plástica Ao & - & 1 & 1 \\
\cline { 2 - 5 } M.T. & plástica + plástica M & - & 1 & 1 \\
\hline
\end{tabular}

MT = mitro-tricuspidea 
LOURES, D. R.; BROFMAN, P. R.; RIBEIRO, E. J.; ROSSI, P. R. F.: PEREIRA, M. A. A.; KRICHENKO, A.; BUENO. R. R. L.; VARELA, A.; BAUER, V.; AMORIM, M. J. F.; LINHARES, L. J. C.; SEEGMULLER, E. F.; MOZACHI, N. - Pode a correção cirúrgica de cardiopatias pediátricas e congênitas conviver com baixa mortalidade?: revisão de 10 anos de experiência com 1088 cirurgias. Rev. Bras. Cir. Cardiovasc., 2(1):32-41, 1987.

TABELA 7

TRATAMENTO CIRÚRGICO DAS VALVOPATIAS ASSOCIADAS A CARDIOPATIAS CONGENITAS EXPERIENCIA DE 1985 E 1986

\begin{tabular}{|c|c|c|c|c|c|c|}
\hline № & DATA & IDADE & NOME & PATOLOGIA & CIRURGIA & EVOL. HOSPIT \\
\hline 1 & 21.1 .85 & 32 & M.N.C. & $\mathrm{IM}+\mathrm{CIA}$ & Fech. CIA + anel Carpentier & OK \\
\hline 2 & 21.3 .85 & 50 & R.A.S. & $\begin{array}{l}\text { Rot. seio } \\
\text { Vals. (v.Ao) }\end{array}$ & Troca mec. V.Ao & OK \\
\hline 3 & 10.12 .85 & 5 & L.R. & IM - cong. & Troca mec. & OK \\
\hline 4 & 18.9 .86 & 24 & R.J. & $\begin{array}{l}\text { EAo Cong. } \\
\text { Calc. }\end{array}$ & $\begin{array}{l}+ \text { AVSVE }=\mathrm{T} \text {. Manoughian } \\
\text { troca mec. }\end{array}$ & OK \\
\hline 5 & 24.11 .86 & 25 & T.C.R. & DLAo + PCA & troca mec. Ao + SS PCA & OK \\
\hline 6 & 20.5 .85 & 13 & H.A. & $\begin{array}{l}\mathrm{ClV}+\mathrm{ISA}+\mathrm{IP}) \\
(\mathrm{EB})\end{array}$ & $\begin{array}{l}\text { Fech. CIV + Dupla troca } \\
\text { Ao e P - mec. e PB }\end{array}$ & OK \\
\hline
\end{tabular}

$\mathrm{IM}+\mathrm{ClA}=$ insuficiência mitral \% comunicação interatrial

Rot. seio Vals. $($ v.Ao) $=$ rotura do seio de Valsalva da valva aórtica

IM. Cong. - insuficiência mitral congênita

DLAo + PCA - dupla lesão aórtica + permanência de canal arterial

$\mathrm{CIV}+\mathrm{IA}+\mathrm{IP}(\mathrm{EB})$ - fechamento comunicação

interventricular + insuficiência aórtica + endocardite

infecciosa bacteriana

TABELA 8

\section{TRATAMENTO CIRÚRGICO COM CARDIOPATIAS PEDIATRICAS (15 ANOS) E CONGENITAS EXPERIENNCIA DE 1985 E 1986}

\begin{tabular}{|c|c|c|c|c|c|c|c|}
\hline \multicolumn{2}{|c|}{ CARD. } & ANO & 1 ANO & $1.4 \mathrm{~A}$. & 4-15 A. & $15 \mathrm{~A}$. & TOTAL \\
\hline \multirow{4}{*}{ C/CEC } & Cian. & 85 & - & $3 / 2$ & 7i- & 3- & $13 / 2$ \\
\hline & & 86 & $1 / 1$ & $9 /-$ & $8-$ & $2-$ & $20 / 1 \quad 33 / 3$ \\
\hline & Acian. & 85 & - & $3 /-$ & 19 - & $12 / 1$ & $34: 1$ \\
\hline & & 86 & $4 / 1$ & $7 /-$ & $28-$ & $9 /-$ & $41 / 1 \quad 75 / 2$ \\
\hline \multirow{5}{*}{ S/CEC } & Cian. & 85 & $5 / 1$ & $1 /-$ & - & - & 611 \\
\hline & & 86 & 82 & 61 & $1 /-$ & - & $15 / 3 \quad 21 / 4$ \\
\hline & Acian. & 85 & 3- & - & $11 / 1$ & $7 /-$ & $20 / 1$ \\
\hline & & 86 & $8 / 1$ & 4-- & $15-$ & $3 /-$ & $29 / 1 \quad 50 / 2$ \\
\hline & & & $\begin{array}{c}29,6 \\
(20,7 \%)\end{array}$ & \begin{tabular}{|c|}
$33 / 3$ \\
$(Q, 1 \%)$
\end{tabular} & $\begin{array}{c}81 / 1 \\
(1,20 \%)\end{array}$ & \begin{tabular}{|c|}
$36 / 1$ \\
$(2,8 \%)$
\end{tabular} & $\begin{array}{l}179 / 11 \\
(6,1 \%)\end{array}$ \\
\hline
\end{tabular}

CEC - circulaçăo extracorpórea SICEC - sem circulaçáo extracorpórea C CEC - com circulaçâo extracorpórea

Qualquer falha, no atendimento de uma arritmia, sangramento, alteração metabólica, insuficiência respiratória, mau débito cardíaco, pode ser fatal, justificando uma postura agressiva do cirurgião e do intensivista pediátrico, no atendimento do pós-operatório.

A tetralogia de Fallot foi a cardiopatia mais freqüente, neste trabalho, representando $13,1 \%$ da experiência global e 76,2 das cardiopatias congênitas cianóticas. A tendência cirúrgica na tetralogia de Fallot é realizar a correção definitiva e num único tempo. A nossa experiência demonstra, nitidamente, este posicionamento, pois $89,1 \%$ dos casos foram corrigidos definitivamente. Quando analisadas as correções em pacientes acima de 1 ano de vida, esta tendência se acentua (96,2\%). A mortalidade hospitalar global, na tetralogia de Fallot, foi de $12,6 \%$ e foi inversamente proporcional à idade, isto é, quanto menor a idade, maior o número de óbitos. Houve uma nítida queda da mortalidade, após o 4. ano de vida. Embora se tenha conhecimento de que a cirurgia da tetralogia de Fallot, em adultos, envolva riscos com o desenvolviento da circulação colateral aortopulmonar, de saturação arterial, aumento da viscosidade sangüínea e comprometimento da função ventricular direita, na nossa experiência, os resultados da correção na faixa etária acima de 15 anos também foram favorávis. LIMA et alii $^{9}$, em 1985, consideram, revisando a experiência do Bromptom Hospital de Londres, que o manuseio cirúrgico, em adulto com cardiopatias congênitas cianóticas, pode ser acompanhado de baixa mortalidade. No tratamento cirúrgico da tetralogia de Fallot, alguns fatores devem ser analisados, para melhor definir a escolha de uma correção definitiva, ou paliativa, a saber: idade, severidade dos defeitos clássicos e presença de defeitos associados, Segundo BINET ${ }^{1}$, a correção cirúrgica de crianças nos primeiros meses, com CEC, incorre em problemas de hemodiluição severa, pois há 
LOURES, D. R.: BROFMAN, P. R.: RIBEIRO, E. J.; ROSSI, P. R. F.; PEREIRA, M. A. A.; KRICHENKO, A.; BUENO, R. R. L.; VARELA, A.; BAUER, V.: AMORIM, M. J. F.: LINHARES, L. J. C.; SEEGMULLER, E. F.: MOZACHI, N. - Pode a correção cirúrgica de cardiopatias pediátricas e congênitas conviver com baixa mortalidade? revisão de 10 anos de experiência com 1088 cirurgias. Rev. Bras. Cir. Cardiovasc., 2(1):32-41, 1987.

uma substituição do volume sangüineo em 3 ou 4 vezes a sua volemia. O líqüído empregado como perfusato ideal seria o sangue, mas, mesmo este, tem anticoagulante, plaquetas destruídas e pode levar a acidose metabólica, alterações pulmonares e modificaçōes no comportamento imunológico Binet acredita que as crianças de baixo peso e operadas com CEC, numa perfusão prolongada, têm uma grande chance de desenvolver, no pós-operatório imediato, baixo débito cardíaco e membrana hialina.

HEGERTY et aliir , em 1985, revisando 291 crianças com cardiopatias congênitas que morreram no 1 : ano de vida, concluíram que o prognóstico cirúrgico está intimamente ligado à complexidade anatômica. Acrescentam que outros fatores podem participar do resultado cirúrgico final, tais como, estudo pré-operatório inadequado, cirurgia com técnica e momento errados. Concluem que a cirurgia, nestas condições, pode antecipar a mortalidade.

MILLIKAN et alii ${ }^{10}$, em 1986, revisando 105 casos de atresia pulmonar com comunicação interventricular, na Mayo Clinic, entenderam que, antes de definir o tipo de correção a ser aplicado, deve a anatomia cirúrgica ser observada com extremo cuidado e concluem que a cirurgia em dois estágios é uma técnica que pode apresentar elevado sucesso e deve ser aplicada quando o estudo anatômico revelar-se complexo e desfavorável para a correção definitiva num único tempo.

A definição anatômica correta dos defeitos é fundamental para o cirurgião e a ecocardiografia mono e bidimensional e o cateterismo cardíaco são importantes, neste sentido.

Compreendemos que o emprego da CEC nos primeiros meses de vida tem inconvenientes, que uma anatomia desfavorável pode dificultar a correção definitiva, que a cirurgia em dois tempos pode ter resultados mais favoráveis e, também, que a posição dogmática de corrigir num único tempo pode ser errada.

Baseados neste raciocínio, tomamos a deliberação de, nos casos de tetralogia de Fallot em pacientes com menos de 1 ano de idade, realizar, preferencialmente, cirurgias paliativas. Noventa por cento de nossos casos sofreram anastomose sistêmica pulmonar, nesta faixa etária.

Por outro lado, algumas malformações impõem uma correção definitiva e o emprego da CEC, ainda nos primeiros meses de vida. Entre estes defeitos, estão a drenagem anômala total das veias pulmonares e o defeito do septo átrio-ventricular total.

OELERT et alii ${ }^{11}$, em 1986, chamam a atenção para que, na DATVP, o procedimento corretivo é a única chance de sobrevivência e que $67 \%$ dos casos operados, no grupo de Hannover, foram antes de 3 meses de vida. A mortalidade global desta experiência foi de $23 \%$, em 53 casos operados. Os mesmos autores enfatizam que a anatomia desfavorável e a hipertensão pulmonar foram consideradas como fatores de incremento na mortalidade. A correção do defeito do septo atrioventricular total é outra das malformações onde a cirurgia definitiva se aplica. Como as manifestações clínicas surgem precocemente, a correção com auxílio da CEC se impõe, nos primeiros meses de vida. Os resultados cirúrgicos vão depender da idade, da anatomia da via de saída do ventrículo esquerdo e do grau de hipertensão pulmonar ${ }^{6}$. $\mathrm{Na}$ nossa experiência, a correção do defeito do septo AV parcial não mostrou mortalidade e a DSAV total, $41,7 \%$. Nos dois últimos anos, foram corrigidos $50 \%$ dos casos de DSAVT, também sem mortalidade.

SANTOS et alii ${ }^{2}$ preconizam o enchimento da cavidade esquerda durante a avaliação transoperatória no DSAV total e acreditam que é maneira prática de identificar as proporções das cavidades ventriculares a correta localização da divisão valvar e a aproximação do parche.

Segundo EBELS et alii ${ }^{6}$ (1986), o trato da saída do ventrículo esquerdo é uma importante estrutura a ser avaliada com maior interesse pelos cirurgiōes, nos casos de DSAV total.

Não existe, ainda, qualquer substituto valvular ideal, especialmente para crianças ${ }^{3,4,5}$.

$\mathrm{Na}$ nossa experiência com valvopatias em faixa pediátrica, 120 casos foram operados e a atitude conservadora foi possível em 55 (1\%). Nos 4 primeiros anos deste estudo, a valva mitral foi conservada em $38,7 \%$ e, nos últimos, em $66,1 \%$. Este aumento na conservação cirúrgica da valva mitral foi somente possível após o retorno de um de nossos cirurgiōes associados, de seu aprendizado no Hospital Broussais, França, com o Dr. Alain Carpentier.

O emprego de novas técnicas de plástica para a conservação da valva mitral tem melhorado o prognóstico cirúrgico e a morbidade (BROFMAN ${ }^{2}$, 1986).

A mortalidade global de 120 valvopatias em fase pediátrica foi de $6,7 \%$, sendo $13,2 \%$ nos primeiros anos e apenas $1,6 \%$ nos últimos 5 anos. 
LOURES, D. R.; BROFMAN, P. R.; RIBEIRO, E. J.; ROSSI, P. R. F.; PEREIRA, M. A. A.; KRICHENKO. A.; BUENO, R. R. L.; VARELA, A.; BAUER, V.; AMORIM, M. J. F.; LINHARES, L. J. C.: SEEGMULLER, E. F.; MOZACHI, N. - Pode a correção cirúrgica de cardiopatias pediátricas e congênitas conviver com baixa mortalidade?: revisāo de 10 anos de experiência com 1088 cirurgias. Rev. Bras. Cir. Cardiovasc., 2(1):32-41, 1987.

Observamos que os resultados cirúrgicos com as valvopatias pediátricas e congênitas foram melhores, nos últimos anos, e em especial em 1985 e 1986. O levantamento estatístico destes dois anos, quando analisados em grupos etários, mostrou que a mortalidade, no 1: ano, permaneceu elevada, com $20,7 \%$, diminuindo nas outras idades, sendo $3,0 \%$ nas idades acima dos 4 anos. A correção cirúrgica em adultos apresentou, também, resultados favoráveis $(2,8 \%)$.
Diversos fatores contribuíram para estes resultados, tais como: melhor preparo pré-operatório; melhor identificação anatômica; indicação cirúrgica correta; escolha da técnica certa; proteção miocárdica completa; melhores condiçōes de pós-operatório, grupos de médicos e enfermagem interessados com o pós-operatório imediato pediátrico e maior experiência cirúrgica.

\begin{abstract}
RBCCV
LOURES, D. R. R.; BROFMAN, P. R.; RIBEIRO, E. J.; ROSSI, P. R.; PEREIRA, M. A. A.; KRICHENKO, A.; BUENO, R. R. L.; VARELA, A.; BAUER, V.; AMORIM, M. J.; LINHARES, L. J. C.; SEEGMULLER, E. R.; MOZACHI, N. - Can surgical correction of congenital heart diseasse go along with low mortality?: 10 year experience in 1088 surgeries. Rev. Bras. Cir. Cardiovasc., 2(1):32-41, 1987

ABSTRACT: From November 1976 to November 1986, 1088 cardiac surgeries were performed in patients younger than fifteen years old. In 670 cases we used extracorporeal circulation, and in 418 we performed surgery without extracorporeal circulation. One hundred and eleven $(10.2 \%)$ patients were reoperated. One hundred and twenty $(11.1 \%)$ patients had acquired valvular heart disease. The total hospital mortality was $10.4 \% ; 11.2 \%$ after extracorporeal circulation and $9.1 \%$ without it. We observed a decrease in the mortality rate in the years 1985 and 1986 , corresponding to $6.8 \%$ and $5.7 \%$, respectively. During this period (1985-1986) 179 patients were operated upon; 54 had cianotic congenital heart disease and 7 died (12,9\%); 125 had cianotic congenital heart disease and 2 died $(1.6 \%)$. The highest mortality rate occured in the first year of life $(20.7 \%)$ while the lowest mortality was observed after the fourth year. Factors which influenced this decrease in mortality included: more frequent indication of palliative surgery in the first year of life; careful analysis of surgical anatomy; precise definitions of the moment of intervention and surgical techniques; better myocardial protection; physical and medical conditions in the post-operative phase and greater surgical experience.
\end{abstract}

DESCRIPTORS: heart disease, pediatric, surgery; heart disease, congenital, surgery.

\section{REFERÊNCIAS BIBLIOGRÁFICAS}

1 BINET, J. P. - The surgery of congenital heart disease in children in 1985. Acta Cardiol., 40 (6): 579-588, 1985.

2 BROFMAN, P. R. - Cirurgia conservadora da valva mitral em pacientes jovens: técnica cirúrgica e resultados. São Paulo, 1986. (Dissertação de Doutorado - Faculdade de Medicina da Universidade de São Paulo).

3 BROFMAN, P. R. - Estudo da evolução de pacientes jovens portadores de bioprótese de dura-máter em posição mitral. São Paulo, 1980. (Dissertação de Mestrado - Faculdade de Medicina da Universidade de São Paulo).

4 BROFMAN, P. R.; CARVALHO, R. G.; RIBEIRO, E. J.; ALMEIDA, R. S.; COELHO, A.; LOURES, D. R. R. - Dura-mater bioprostheses in young patients. In: COHN, L. \& GALLUCCI, V. eds. Cardiac bioprostheses. New York, York Medical Books, 1982. p.266-272.

5 BROWN, J. W.; DESCHENER, W. R.; COOPER, G. L.; KING, H. - Aortic and mitral valve replacement in children: a 21 year experience. Cardiol. Clin., 3(3): 479-492, 1985.
6 EBELS, T.; YEN HO, S.; ANDERSON, R. H.; MEIJBOOM, E. J.; EI JGELAAR, A. - The surgical anatomy of the left ventricular outflow tract in atrioventricular septal defect. Ann. Thorac. Surg., 41 (5): 483-488, 1986.

7 HEGERTY, A.; ANDERSON, R. H.; YEN HO, S. - Congenital heart malformations in the first year of life: a necropsy study. Br. Heart J., 54 (6): 583-592, 1985.

8 LILLEHEI, C. W.; VARCO, R. L.; COHEN, M.; WARDEN, H. E.; PATTON, C.; MOLLER, J. H. - The first openheart corrections of ventricular septal defect, atrioventricular communis, and tetralogy of Fallot utilizing extracorporeal circulation by cross-circulation: a 30 year follow-up. Ann. Thorac. Surg., 41 (1): 4-21, 1986.

9 LIMA, R. C.; FIRMIN, R. K.; PILLAI, R.; LENNOX, S. C.; CLELAND, W. P.; PANETH, M.; LINCOLN, C. R. Surgical management of adults with cyanotic congenital heart disease. Arq. Bras. Cardiol., 45 (3): 175-180, 1983.

10 MILLIKAN, J. S.; PUGA, F. J.; DANIELSON, G. K.; SCHAFF, H. V.; JULSRUD, P. R.; MAIR, D. D. Staged surgical repair of pulmonary atresia, ventricular septal defect, and hypoplastic, confluent pulmonary artery. J. Thorac. Cardiovasc. Surg., 91 (6): 818-825, 1986. 
LOURES, D. R.; BROFMAN, P. R.; RIBEIRO, E. J.; ROSSI, P. R. F.; PEREIRA, M. A. A.; KRICHENKO, A.; BUENO, R. R. L.; VARELA, A.; BAUER, V.; AMORIM, M. J. F.: LINHARES, L. J. C.; SEEGMULLER, E. F.: MOZACHI, N. - Pode a correção cirúrgica de cardiopatias pediátricas e congênitas conviver com baixa mortalidade?: revisâo de 10 anos de experiência com 1088 cirurgias. Rev. Bras. Cir. Cardiovasc., 2(1):32-41, 1987

11 OELERT, H.; SCHÄFERS, H. J.; STEGMANN, T.; KALLFELZ, H. C.; BORST, H. G. - Complete correction of total anomalous pulmonary venous drainage: experience with 53 patients. Ann. Thorac. Surg., 41 (4): 392-394, 1986.

12 SANTOS, A.; BOUCEK, M.; BUTTENBERG, H.; VEASY, G.; ORSMOND, G.; McGOUGH, E. - Repair of atrioventricular septal defects in infancy. J. Thorac. Cardiovasc. Surg., 91 (4): 505-510, 1986.

\section{Discussão}

DR. FERNANDO A. LUCCHESE

Porto Alegre, RS

Foi revisada a casuística do Serviço do autor, compreendendo 1088 cirurgias, divididas entre cirurgias de valvopatias adquiridas ( $<15$ anos) e cirurgias de cardiopatias congênitas (todas as idades). A mortalidade global foi de $10,4 \%$; como era de se esperar, a mortalidade foi significativamente menor em crianças operadas nos últimos anos (1985 e 1986) e em pacientes maiores ( $>4$ anos). Em relação a patologias específicas, como o DSAV, há inclusão no grupo dos casos de CIA ostium primum, os quais, sendo em maior número, reduziram a mortalidade do DSAV de $41,7 \%$ para $17,2 \%$, já que não houve nenhum óbito, no grupo das CIA. O autor mostra, contudo, que a mortalidade dos seis últimos casos de DSAV (comp.) foi zero, nos últimos 2 anos. Em relação às valvopatias, a mortalidade (abaixo de 15 anos) foi de $6,7 \%$ (sendo $13,2 \%$ nos primeiros anos e 1,6\% nos últimos 5 anos). Em relação às cardiopatias congênitas cianóticas, tetralogia de Fallot foi a mais prevalente $(13,1 \%$ do total de casos) e cerca de $76,2 \%$ das cardiopatias congênitas cianótica operadas. A tendência do Serviço é correção em um estágio. Novamente, a mortalidade foi indiretamente proporcional à idade. Aqui, o autor evoca a experiência do Brompton, sugerindo que o manuseio do adulto com cardiopatia cianótica pode ser acompanhado de baixa mortalidade. Fato de importância discutível, visto que há nítida tendência, nos dias de hoje, para tratamento cirúrgico definitivo em crianças menores. Do ponto de vista da discussão, acho que devem ser enfatizados os seguintes aspectos: 1) mortalidade global está dentro dos limites encontráveis na literatura; 2) há possibilidade de se operarem, com mortalidade, crianças com cardiopatias congênitas e até correção definitiva precoce. Para tal, concorrem vários aspectos, a saber (genericamente) (Slide): A) chegada precoce, ao hospital, dos pacientes em melhores condições, para maior esclarecimento dos pediatras e maior facilidade de obtenção de centros de referência, para internação; B) efeito do ecocardiograma bidimensional (ou da diminuição dos cateterismos em crianças pequenas) sobre a mortalidade cirúrgica dos pacientes (tanto em relação às cirurgias corretivas ou paliativas). Aqui convém citar a experiência do Brompton com drenagem anômala pulmonar total (1973-1984), que mostra nitidamente, que a mortalidade cirúrgica caiu vertiginosamente, sendo o único fator para tal o não uso do estudo hemodinâmico nos pacientes operados após 1976 . Nos últimos 20 casos consecutivos, não houve mortalidade. (Ref. preliminary report). C) efeito "Lázaro" - foi ítem no qual o cirurgião baseou-se para mostrar a melhoria de sua estatística. Enfatizamos a importância da curva de aprendizado, na melhoria da técnica cirúrgica. D) melhores condições de perfusão com uso adequado de parada circulatória e baixo fluxo. E) condições de pós-operatório que melhoraram na última década (UTI pediátrica, enfermagem treinada, meIhoria na ventilação mecânica, nutrição parenteral, melhor preparo do staff médico que cuida dos pacientes). 3) Discutimos a tendência do autor de manter pacientes por mais tempo antes de indicar cirurgia, pois existem problemas em relação a esta abordagem. Por exemplo: pacientes severamente cianóticos podem desenvolver complicações vasculares cerebrais ou lactentes cianóticos que parecem ter pior desempenho psicomotor se não corrigidos antes de 6 meses. Enfatizamos, no entanto, a tentativa de se pouparem valvas com procedimentos de plastia, especialmente em crianças menores, tentando evitar próteses. Estes, ao meu ver, são os principais aspectos a serem enfocados para responder positivamente à pergunta "se é possivel operar cardiopatias congênitas com baixa mortalidade." Mortalidade de TGV com septo intacto, de Jan Quaegebeur (J. Thorac. Cardiovasc. Surg., 2:361-384, 1986), que é de $0,3 \%$, para neonatos.

\section{DR. RICARDO DE CARVALHO LIMA Recife, PE}

Desejo, inicialmente, cumprimentar o Dr. Danton e seus colaboradores pela excelência de seu trabalho. A seguir, apresento nossa experiência, durante o período de dezembro de 1984 a março de 1987. Noventa e seis pacientes com idade abaixo de 15 anos foram operados, no Hospital dos Servidores do Estado de Pernambuco e na Unidade de Tratamento Cárdio-Torácico do Real Hospital Português do Recife. A cardiopatia adquirida esteve presente em 11 crianças e, nas 85 restantes, os defeitos foram congênitos. A idade variou de $1 \mathrm{dia}$ a 15 anos e o peso, de 2.800 a $47 \mathrm{Kg}$. Plastia 
LOURES, D. R.; BROFMAN, P. R.: RIBEIRO, E. J.: ROSSI, P. R. F.: PEREIRA, M. A. A.: KRICHENKO. A.: BUENO. R. R. L.: VARELA, A.; BAUER, V.: AMORIM, M. J. F.: LINHARES, L. J. C.: SEEGMULLER. E. F.: MOZACHI. N. - Pode a correçâo cirúrgica de cardiopatias pediátricas e congênitas conviver com baixa mortalidade?: revisão de 10 anos de experiência com 1088 cirurgias. Rev. Bras. Cir. Cardiovasc., 2(1):32-41, 1987

da valva mitral foi realizada em 2 pacientes, troca valvar mitral em 5 , com dois óbitos $(40,0 \%)$; troca valvar aórtica em 2 e dupla troca $(\mathrm{M}+\mathrm{Ao})$ em 2 , com um óbito $(50,0 \%)$. A mortalidade, neste grupo, foi de $27,3 \%$ (3 pacientes). No grupo das cardiopatias congênitas corrigidas com auxílio de circulação extracorpórea (CEC), a comunicação interatrial esteve presente em 6 , comunicação interventricular em 12, drenagem anômala total das veias pulmonares em 1, estenose pulmonar infundíbulo-valvar em 4, tetralogia de Fallot em 2 e dupla via de saída do ventrículo direito em 3 . A mortalidade, neste grupo, foi de $3,6 \%$ (1 paciente com DATVP). As cardiopatias operadas sem auxílio de CEC apresentaram mortalidade de $12,3 \%$ (7 pacientes). Vinte e dois pacientes tinham persistência do canal arterial; 3 tinham coarctação da aorta; 1 , estenose pulmonar valvar e 4, comunicação interventricular (três óbitos, após cerclagem da artéria pulmonar). As cardiopatias de hipofluxo pulmonar foram tratadas com shunt sistêmico-pulmonar (Blalock-Taussig em 25, três óbitos, Waterston em 2, com um óbito). O melhor resultado, no grupo das cardiopatias congênitas, deveu-se a um melhor conhecimento da morfologia cardíaca, bem como melhores cuidados pós-operatórios. Neste grupo, os pacientes com cardiopatias adquiridas foram operados em fase avançada da cardite reumática, colaborando, assim, para um significante aumento da mortalidade.

\section{DR. LOURES}

(Encerrando)

Aos comentadores Dr. Lucchese e Dr. Lima, meus agradecirnentos. Dr. Lucchese coloca em dis- cussão o fato suposto de nossa tendência em evitar indicação cirúrgica definitiva em recém-natos e em crianças com menos de 6 meses de idade e argumenta que pacientes severamente cianóticos podem desenvolver complicações vasculares cerebrais com baixo desempenho psicomotor. Em resposta a esta colocação, gostariamos de ponderar três aspectos: primeiro, as crianças com menos de 6 meses e com indicação de cirurgia, geralmente são portadoras de cardiopatias complexas, anatomia desfavorável, baixa superfície corpórea e imaturidade orgânica; segundo, as tentativas, em nossas mãos, de correção definitiva, nesta faixa etária, evoluíram com elevada mortalidade e, finalmente, o emprego de uma cirurgia paliativa, como primeiro tempo da correção definitiva, antecedida de cuidados clínicos para o equilíbrio metabólico e uso de drogas betabloqueadoras, prostaglandina, indometacina e balão de Rashkind, definitivamente diminuíram nossa mortalidade. Temos reservada a cirurgia definitiva, nesta faixa etária (menos de 6 meses), para as malformações onde as paliações não se aplicam, ou têm maus resultados, tais como a drenagem anômala total das veias pulmonares e o defeito do septo atrioventricular completo. Em resposta ao Dr. Lima, gostaria de ressaltar dois aspectos: a) o emprego da ecocardiografia e as melhores condiçōes no atendimento do pós-operatório imediato foram dois avanços importantes na melhoria dos resultados cirúrgicos nas cardiopatias congênitas; b) a atitude cirúrgica conservadora e a indicação de operações mais precoces com melhores condições miocárdicas influenciaram nossas estatísticas, nestes últimos 5 anos $(1,6 \%)$. 